\title{
Comunidades de práctica y alfabetización informacional
}

\author{
Communities of practice and information literacy
}

\author{
Guadalupe VegA (1), Álvaro QUIJANo (2) \\ Biblioteca Daniel Cosio Villegas, Colegio de México, A. C., Camino al Ajusco n. ${ }^{\circ} 20$. \\ Pedregal de Santa Teresa; 01000 México, D. F., (1) guvega@colmex.mx (2) quijano@colmex.mx.
}

\begin{abstract}
Resumen:
Se analiza el concepto de comunidades de práctica como un espacio de aprendizaje en donde individuos con diferentes niveles de conocimientos y experiencias analizan y solucionan problemas que emergen de situaciones cotidianas; al tiempo que negocian significados, reflexionan para entenderse, planifican e implementan las acciones pertinentes. Acorde con este concepto, se propone que la Alfabetización Informacional se promueva considerando la interacción y la participación informada de sujetos que participan en diferentes comunidades de práctica. La propuesta tiene como marco la perspectiva sociocultural del aprendizaje, en particular se hace referencia a la Teoría de la Actividad, y se ilustra su aplicación con un taller dirigido al personal bibliotecario de una biblioteca académica en México.
\end{abstract}

Palabras clave: Comunidades de práctica. Apropiación. Competencias informacionales. Alfabetización informacional. Aprendizaje significativo.

\section{Concepto de Comunidades de Práctica}

El término comunidades de práctica [CdP] fue acuñado por Lave y Wenger (1991), a partir de los estudios sobre el aprendizaje situado. Este tipo de aprendizaje se conceptualiza como aquél que permite vincular las experiencias reales que suceden en diferentes contextos con el aprendizaje escolar.

Entre las principales características de las CdP se destacan las siguientes: a) la coexistencia de relaciones de armonía y conflicto; b) el interés de sus miembros para realizar actividades de manera conjunta; c) los flujos adecuados y ágiles de información y difusión de la innovación; d) la existencia de un ambiente adecuado que permite la discusión de problemas sin mayor preámbulo; e) la existencia de un código no escrito de puntos en común entre todos sus integrantes; f) la conciencia sobre las habilidades y conocimientos de todos los miembros que conforman la comunidad; g) la comunicación entre sus miembros, que se da mediante una jerga común y términos específicos; y h) el

\begin{abstract}
The concept of communities of practice is analyzed as a learning space where individuals with different knowledge levels and experiences analyze and solve problems emerging from daily situations. At the same time that they negotiate meanings, the reflect together, plan and develope the pertinent actions. According to this concept, it is proposed that Information Literacy is promoted considering the interaction and informed participation of subjects that belong to different communities of practice. The proposal has a frame the sociocultural perspective of learning, in particular the Theory of Activity, and its applications is exemplified with a workshop addressed to the library personal of a Mexican academic library.
\end{abstract}

Keywords: Communities of practice. Appropiation. Information competences. Information literacy. Significative learning.

compartir un discurso común sobre la visión del mundo (Wenger 1998/2001).

La CdP es conceptualizada como un espacio de aprendizaje, negociación, significado e identidad, en donde los sujetos que participan se otorgan mutuamente un sentido de pertenencia a la comunidad y una participación "legítima", que puede ser central o periférica (Wenger, 1998). En las CdP los integrantes interactúan y generan normas y relaciones; además de que crean y comparten recursos comunes, rutinas y artefactos.

Para Loyarte y Rivera (2007) existen aspectos críticos que inciden en la implantación de las CdP, entre los que destacan: a) el tipo de conocimiento que se desea compartir; b) el grado de conexión e identidad que existe entre los integrantes de la comunidad; y c) la integración en intercambio de conocimiento y trabajo. Otro aspecto importante que los autores señalan es el valor de la teoría de Wenger para evaluar la integración de las CdP dentro de las organizaciones, la cual se fundamenta esencialmente en cuatro aspectos: El significado (aprendizaje 
como experiencia), la práctica (aprender - know how), la comunidad (pertenencia) y la identidad.

Para Evans y Niemeyer (2004) el concepto de $\mathrm{CdP}$ ha evolucionado hacia las comunidades de aprendizaje centradas en la práctica [CdAP]. En particular, Niemeyer (2006) analiza el concepto de CdAP para presentar una propuesta de inserción de los alumnos de educación superior en el mercado laboral. La idea es que los alumnos pueden aprender de mejor manera cuando se involucran activamente en la solución de problemas reales relacionados con las actividades de una comunidad a la cual ellos van a pertenecer eventualmente. Desde nuestro punto de vista, estas actividades, son las que permiten el intercambio de significados y dan sentido de identidad a un grupo. Este intercambio de significados requiere que el sujeto, integre sus habilidades, conocimientos y actitudes para participar, compartir información $\mathrm{y}$, así, ayudar a cohesionar la comunidad.

Adicionalmente, esta forma de aprender en comunidades se ha analizado desde el concepto de "aprendizaje situado" (Lave y Wenger, 1991), que reconoce que el aprendizaje es social por naturaleza y se centra en cómo los aprendices o novatos se insertan en una comunidad de práctica, pasando de una participación periférica legitimada a una participación completa y muy cercana a los expertos que se mueven en el centro de la comunidad, ayudando 0 monitoreando a los nuevos participantes. En contraparte, Lave y Wenger consideran que los espacios áulicos descontextualizan el conocimiento y le quitan historicidad, a pesar de que la escuela misma es un contexto específico en un marco histórico. Para Diaz Bariga (2003, p. 112), el aprendizaje situado puede fomentarse a través de las siguientes estrategias didácticas: a) aprendizaje centrado en la solución de problemas auténticos; b) análisis de casos; c) método de proyectos; d) prácticas situadas o aprendizaje in situ en escenarios reales; e) aprendizaje en el servicio; f) trabajo en equipos cooperativos; g) ejercicios, demostraciones y simulaciones situadas; y h) aprendizaje mediado por las nuevas tecnologías de la información y la comunicación (NTIC).

Otro de los conceptos básicos de las CdP es el de la cognición distribuida, con el que se reconoce que en una comunidad hay actores que cubren diferentes roles y que tienen diferentes niveles de conocimiento y expertise para realizar una actividad. Para Dalkir (2005) los roles que se cubren son:

- Miembros experimentados. Son considerados expertos y fuentes confiables de cono- cimiento. Se encuentran familiarizados con el tema profesional y de la comunidad. Representan la memoria histórica de la CdP y pueden ser consultados por otros miembros.

- Lideres. Dirigen la comunidad y brindan todo el apoyo por su correcta operación.

- Miembros regulares. Llevan a cabo contribuciones regulares dentro de la $\mathrm{CdP}$ e interactúan con otros miembros bajo bases sustentadas.

- Miembros principiantes. Nuevos miembros que se valen de ellos mismos, hasta haber completado el proceso de aprendizaje de la comunidad y sus miembros. Es en estos miembros en donde se centra la participación periférica legítima.

- Visitantes. Personas interesadas en las actividades llevadas a cabo por la comunidad de práctica.

En las organizaciones, las $\mathrm{CdP}$ representan una estructura complementaria, de carácter informal e integradora y cuyo fin es mejorar las tareas diarias de una organización. En ellas, el aprendizaje encuentra un vínculo indisoluble con la práctica y la socialización del conocimiento y la experiencia. Las relaciones que se establecen dentro de la comunidad toman una jerarquía entre los miembros que la conforman; estas relaciones son dinámicas en relación a la evolución de los participantes periféricos a expertos y las múltiples interacciones simétricas y asimétricas entre los diferentes actores.

Gracy (2001) aplicó el concepto de CdP para analizar el trabajo de los archivistas a partir del método de la etnografía, para tipificar a las primeras como "comunidades de individuos que generan, acumulan y conservan evidencia documental" (p. 212). El estudio se basa en el análisis de los procesos y prácticas de los sujetos que construyen, producen, mantienen $y$ utilizan los documentos en los contextos en los que ocurren las actividades. También, incluye un análisis de las percepciones de los aprendices sobre los expertos que organizan y conservan los documentos. Otro estudio en el que se han incluido las CdP es el de Triggs (2004), que se basa en el estudio de una comunidad de abogados, en la cual se identifican tres niveles de comunidades interconectadas, las micro, meso y macrocomunidades. En el estudio se analizaron los diálogos que se establecen entre los investigadores y los practicantes usando las tecnologías de la información.

Davenport (2002) realizó un meta-análisis para el Annual Review of Information Science and Technology. En su estudio, la autora definió a la 
CdP como "un grupo flexible de profesionales, que tienen intereses comunes que interactúan a través de tareas interdependientes que son guiadas por un propósito común dirigido a almacenar el conocimiento de la comunidad" (p.171). En su análisis, Davenport señala que en las $\mathrm{CdP}$ es posible explorar el contexto social y físico en el que los artefactos son utilizados para comprender los roles internos que juegan los objetos. Davenport identifico tres tipos de estudios en las ciencias de la información. El primero se encuentra relacionado con el aprendizaje situado, el segundo con la cognición distribuida y el tercero se refiere a los estudios sobre análisis del discurso. Entre los estudios que menciona se encuentra el análisis del género literario y de la conversación en relación al uso de los tipos de documentos en diferentes comunidades (Yates y Orlikowski, 1992). Davenport incluye un análisis de las comunidades de práctica en el trabajo, en el marco del acceso a la información y el conocimiento como parte de las recompensas por pertenecer a una comunidad, incluyendo el estatus y la reputación. Por último, Davenport analiza los estudios bibliométricos y el análisis de citas como parte de las evidencias que dan cuenta de la pertenencia a una comunidad científica, aunque advierte que más que dirigirse a la recuperación individual de documentos, estos estudios se refieren a las relaciones entre los miembros de una misma comunidad que citan o son citados en la misma.

\section{Marco teórico de las CdP}

El concepto de CdP se deriva de la perspectiva sociocultural, la cual pondera la importancia del "aprendizaje situado" para contextualizar la enseñanza. En esta perspectiva los sujetos se apropian de los artefactos culturales de una comunidad a través de procesos de mediación que permiten comprender y transformar los usos de dichos artefactos. Un artefacto puede ser una herramienta material (computadora, lápiz, etc.) o una herramienta psicológica (signos y símbolos). La mediación se basa en el diseño un sistema de actividades significativas ubicadas dentro de las prácticas de una comupidad

complejidad del sistema de actividades, los pioneros de la perspectiva sociocultural desarrollaron la "teoría de la actividad" (TA), en la cual se desglosan los elementos que están inmersos en dicho sistema. Este sistema es visto de manera holística como un sistema de actividad mediado que comprende al sujeto, sus compañeros de comunidad, las herramientas materiales y psicológicas y los objetos compartidos (Engëstrom, 1987; Wilson, 2008 , p. 120). Daniels propone tres momentos en la evolución de la TA (2001/2003). En el primer momento, se encuentra la propuesta de Vigotsky (1978) que formula el concepto de mediación y lo relaciona con las herramientas psicológicas y el objeto de aprendizaje.



Figura 1. Proceso de Mediación (adaptado de Leontiev, 1993/1977)

En un segundo momento, Leontiev (1993/1977) identifica que la mediación se compone de tres elementos que se interrelacionan: las actividad, las acciones y las operaciones (figura 1).

En el modelo de la figura 1, existen tres niveles jerárquicos de mediación. El primero es el de la actividad el cual se relaciona con los motivos y las metas que establecen los sujetos para conocer un objeto determinado. Las acciones se vinculan con los conocimientos y habilidades de los sujetos para conocer dicho objeto. Por último, las operaciones están asociadas con las condiciones específicas en las cuales se lleva a cabo la actividad, así como con las rutinas y la normatividad que están implicadas (Barab, 
Evans y Baek, 2004). La actividad ocurre en el ámbito grupal y las acciones y la operación en el individual. En un tercer momento, la TA se enri- quece con las aportaciones de Engeström, quien contextualiza la actividad y la integra al concepto de comunidad (figura 2).

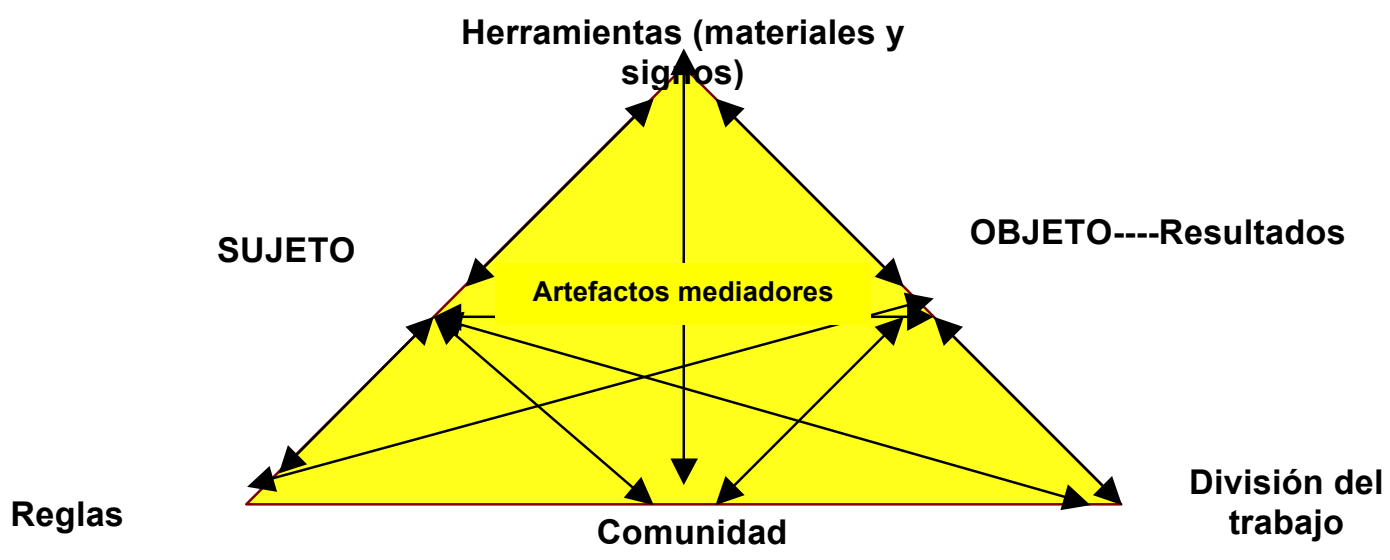

Figura 2. Sistema Humano de Actividad (Engeström, 1987)

En su propuesta, Engeström (1987) afirma que las actividades humanas se realizan en dos planos. El primer plano retoma la propuesta de Vygotsky (1978) y en la segunda integra los procesos de división del trabajo, las reglas y la división del trabajo. Engeström resalta el papel de las contradicciones dialécticas como tensiones estructurales cuya solución es el motor del cambio. En su propuesta, Engeström explicita la importancia de las comunidades en los procesos de aprendizaje y las retoma como parte del proceso de conocer y de aplicar ese conocimiento.

Daniels (2001/2003) señala que los artefactos tienen historicidad y orientación, y que la mediación se basa en la noción de procesos dialécticos en donde se llevan a cabo cambios cualitativos de manera permanente, que es lo que posibilita la transformación y la evolución de los conocimientos a nivel generacional.

En las ciencias de la información la TA ha sido utilizada para comprender el comportamiento informativo desde su significado social en el marco de las comunidades de aprendizaje y de práctica (Foster, 2006; http://www.refworks.com/Refworks/\%7E0\%7E Wilson 2000, 2008). Wilson (2008) reseñó las aplicaciones de la TA en la literatura de aquella disciplina haciendo énfasis en la agenda de investigación de la alfabetización informacional. Wilson también identificó los trabajos de Hjorland (1997), quien aplicó la TA en los procesos de clasificación.
Para Wilson (2008), la comprensión de la TA en las ciencias de la información se observa en cuatro dimensiones: la práctica, la cognitiva, la educativa y la organizacional. En todas ellas se enfatiza que la actividad eje es la bibliográfíca, ya que comprende una integración holística de los datos producidos en diferentes medios y es, en muchos sentidos, un fenómeno social complejo que tienen múltiples representaciones a nivel individual y social (catálogos de bibliotecas, enciclopedias, investigación documental, etc.). En este sentido, la bibliografía implica acciones internas y externas y está influenciada por intereses y metas específicas, además de que responde a contextos histórico-socioculturales específicos.

\section{CdP y Alfabetización informacional}

En los últimos años se ha incrementado la realización de meta-análisis sobre la Alfabetización Informacional (ALFIN), lo que podría ser un indicador de que las reflexiones sobre el tema han llegado a un punto en que se está tratando de buscar patrones y modelos de evolución comprensivos (Bawden, 2001; Rader, 1995, Rendón, 2007; Lau, 2007). En este mismo sentido, podemos afirmar que el concepto de ALFIN ha evolucionado a partir de los aspectos culturales que están presentes en la sociedad de la información (Johnston y Webber, 2005). Entre los aspectos que han incidido en tal evolución podemos distinguir las tendencias educativas centradas en el constructivismo y el cambio constante de las tecnologías de la información y 
la comunicación (TIC). Otro aspecto es el que se relaciona con un incremento en los estudios sobre patrones de conducta y comportamiento en el uso de la información que se han derivado de las teorías de la psicología cognitiva (Wilson, 2000, 2008; Foster, 2006, 2004; Eisenberg, 2004; Bilal, 2002).

En la actualidad se considera que la ALFIN no sólo se refiere a un conjunto de competencias o atributos personales (ALA, 1993), sino que se vincula con las prácticas que permiten a los sujetos de una sociedad usar la información para resolver problemas específicos y, en términos generales, para hacer frente a las situaciones de aprendizaje cotidianas en las que se requiere hacer uso de la información. En este sentido, una persona alfabeta en información sería capaz de identificar la que le es relevante para una actividad determinada, y sabría cómo obtener información del entorno, ya sea que este estructurada o no. Todo ello con el objetivo de tener una participación informada y pertinente en las comunidades en donde se trabaja y aprende. Así, una comunidad es el espacio natural donde se realiza la actividad y es, a su vez, el espacio en donde se aprende y se negocian significados e identidades.

Desde nuestra perspectiva uno de los autores que ha promovido la comprensión de la ALFIN de forma contextualizada es Lloyd (2005), quien identifica tres contextos en donde se desarrollan estas comunidades. El primer contexto es el de la educación básica, cuyo objetivo primordial esta dirigido al aprender a aprender. El segundo contexto es el de la educación superior, el cual se centra en las competencias para usar la información y en la transferencia de los métodos para resolver problemas de información en diferentes escenarios. El tercer contexto es el laboral e implica el reconocimiento de que la información es producida y distribuida socialmente de acuerdo con la estructura organizacional. El autor reconoce que este último escenario no es neutral, ya que hay relaciones de poder que influyen en la distribución de la información en las organizaciones. En este escenario, el aprendizaje es más informal, flexible y problemático, tal y como ocurre en la vida real. En los ambientes laborales, Lloyd considera que hay varias formas de obtener información: por medio de los textos (normas, rutinas, procedimientos); por medio de las relaciones sociales (información valorada por la comunidad, experiencias, etc.); y por medios físicos (observación, manipulación de artefactos, etc.).

A partir de la conceptualización de Lloyd podemos establecer un vínculo con el concepto de comunidades de práctica $(\mathrm{CdP})$. Este vínculo, puede derivarse de dos aspectos fundamentales: el primero es la posibilidad de observar a la bibliografía como un sistema de actividades social y culturalmente significativas, en el sentido de la TA. El segundo es el aprendizaje colaborativo entre los diferentes actores de una $\mathrm{CdP}$, que permite la contextualización sociohistórica de una comunidad a partir de los diferentes tipos de documentos, comunicaciones personales y de la información disponible en el entorno en el que los sujetos de una comunidad desarrollan sus prácticas.

Por su parte, a partir de la propuesta de Wilson (2008) que vincula la ALFIN con la TA, es posible identificar los componentes del sistema de actividad: Wilson establece que el sujeto es la persona que participa en una comunidad a la que se le presenta un problema que puede resolverse con información. En cuanto al objeto, Wilson considera que es el conjunto específico de competencias que son deseables para un grupo de sujetos. Los artefactos pueden ser las computadoras, los tesauros, los índices, los sistemas de clasificación, etc.

En el caso de las reglas y normas, Wilson (2008) las vincula con las normas informales y formales para usar la información y los aspectos éticos de su uso. De manera particular, las vincula con el uso de las convenciones para la citación que permiten construir el aparato crítico de un documento y respetar los derechos de autor. En relación al concepto de comunidad, lo relaciona con el aprendizaje que se puede promover entre pares en diferentes niveles de una organización. El trabajo colaborativo implica la división del trabajo que, inclusive, puede referirse a la división de roles en el proceso de enseñanza-aprendizaje. Por último, el producto comprende el proceso de valoración de la ALFIN en relación a su impacto a largo plazo.

Desde nuestra perspectiva, la TA permite evidenciar la importancia de incluir actividades relevantes asociadas a prácticas reales, así como las reglas de colaboración y de distribución de las acciones en situaciones que son significativas para un grupo de personas. En relación a lo anterior, reseñamos tres casos concretos de contextualización de actividades de comunidades específicas, en donde la actividad planteada se relacionó con procesos bibliográficos.

- Vega (2009) aplicó los elementos de la TA para estudiar a un grupo de alumnos de educación básica e identificar la forma en la que interactuaban en el uso de información en un proyecto escolar donde tenían que incluir las fuentes de información consultadas. 
Entre sus hallazgos encontró que los alumnos usaron tres tipos de fuentes de información: las formales (libros, enciclopedias, etc. en formato impreso o electrónicas); las personas (maestros, alumnos, padres, etc.); y la información que provenía de los programas y ayudas didácticas que se encontraban en el aula de clases. También encontró que los alumnos, al trabajar de manera colaborativa entre pares, mejoraron sus competencias para usar la información y las pudieron aplicar para realizar una investigación escolar.

- Wastawy (2004), en un estudio con alumnos de una universidad, identificó que los estudiantes se agrupaban de manera informal para alcanzar sus metas académicas. Estas agrupaciones informales eran comunidades en las que ellos sentían satisfacción de participar ya que podían establecer sus propias reglas de interacción y determinaban las fuentes de información que eran apropiadas, incluyendo las de menor calidad, pero que les permitían dar respuesta inmediata. Wastawy concluyó que las bibliotecas académicas deberían estar diseñando servicios para atender dichas comunidades, por ejemplo, asignándoles un tutor-bibliotecario que les acompañe en sus procesos de aprendizaje. También propone que se revise la forma como se usan los recursos de información, el uso de literatura gris y la impartición de programas de ALFIN vinculados a eventos de aprendizaje específicos.

- Ortiz, Vega y Quijano (2008) analizaron una CdP de periodistas, con el objetivo de identificar el tipo de fuentes de información que utilizaban y cómo influían, en su uso, el contexto y la demanda laboral. Entre sus hallazgos más importantes se encuentra el reconocimiento de que hay normas en una organización que determinan la cantidad y la calidad de las fuentes de información que utilizan los trabajadores para realizar sus actividades cotidianas.

En los tres casos, correspondientes a CdP diferentes, la ALFIN se incorporó como parte de las acciones dirigidas a la selección, evaluación e integración de la información para diferentes actividades (elaborar una conferencia, realizar una nota periodística o realizar un trabajo escolar) compuestas, a su vez, por diferentes operaciones (analizar la tarea, buscar información, interactuar con bases de datos o informantes clave).

\section{ALFIN en una comunidad de práctica}

El objetivo de este estudio fue analizar la colaboración entre expertos, que actuaron como facilitadores y 62 paraprofesionales, que actuaron como aprendices. El objetivo de esta colaboración fue mejorar el aprendizaje de los últimos en el uso e instrucción sobre el catálogo bibliográfico de una biblioteca académica.

El estudio se basó en la observación y la reflexión de una actividad específica, un taller, Al finalizar el taller, se aplicaron dos cuestionarios. Estos cuestionarios tenían un carácter exploratorio sobre el aprendizaje situado y colaborativo y no fueron pensados para evaluar las competencias en ALFIN. El primer cuestionario buscaba conocer las percepciones de los participantes novatos sobre la colaboración entre los integrantes de la comunidad y sobre su autoevaluación sobre su aprendizaje. El segundo se dirigió a conocer la opinión de los facilitadores sobre su propio desempeño y las interacciones del grupo.

\subsection{Comunidad de Práctica}

Esta comunidad se compone de dos grupos de personas provenientes de las diferentes áreas de la biblioteca y que, como parte de sus funciones, han venido proporcionando instrucción bibliográfica sobre el uso del catálogo en línea a los usuarios finales a través del servicio del "Módulo de Referencia" en una biblioteca académica. En este sentido, la comunidad ha existido por mucho tiempo y reviste alguna de las características que se han descrito anteriormente para las $\mathrm{CdP}$. Por ejemplo, hay relaciones de armonía y conflicto entre sus integrantes; hay un interés para la realización de la actividad conjunta, con la conciencia de que ésta es importante; existe un código tácito de comportamiento entre los integrantes y se comparte una visión común del mundo.

Para efectos de la tarea, se tomaron los dos grupos mencionados que narramos como grupos 1 y 2, y se formó un tercero, el cual tenía la encomienda de documentar el proceso. Estos grupos fueron:

- Grupo 1. Formado por 62 paraprofesionales que utilizan el catálogo en línea para realizar sus labores cotidianas (selección, precatalogación, atención del módulo de información, etc.)

- Grupo 2. Formado por profesionales que diseñan e implementan los cursos y talleres sobre el uso del catálogo.

- Grupo 3. Formado por los sujetos que realizaron el análisis de los procesos. 
Aunque esta comunidad habia venido recibiendo capacitación sobre el uso del catálogo, se observó que faltaba fortalecer el sentido de identidad, mejorar los flujos de información y tomar conciencia colectiva sobre los conocimientos y experiencias de sus integrantes para proporcionar instrucción sobre el uso del catálogo. Se podría decir que cada uno de ellos resolvía los problemas de manera individual, sin conocer cómo estaban proporcionando instrucción sus demás compañeros y sin socializar los problemas de información relacionados con el uso del catálogo que podría tener el usuario final. Es decir, sin una conciencia colectiva de cuáles eran las acciones y las operaciones más apropiadas en relación con la propia comprensión sobre la búsqueda, selección y manipulación de la información en el catálogo en línea.

Al darse el cambio de interfaz del catálogo en línea, que suponía una manera diferente de interactuar con la base de datos, se presentó la oportunidad de revisar y mejorar integralmente las habilidades y conocimientos sobre el uso de esta herramienta. Acorde con lo anterior, el Grupo 3 diseñó un proceso de ALFIN para promover la resolución colaborativa de problemas reales relacionados con el inminente cambio tecnológico del catálogo en línea.

La situación sirvió para retomar la información relevante sobre el uso del catálogo de cada uno de los integrantes de la CdP para, a partir de ella, construir una noción de identidad que les permitiera compartir experiencias y conocimientos. En este sentido, el proceso de ALFIN tenía por objetivo fortalecer las habilidades y conocimientos para buscar, seleccionar y manipular la información del catálogo de los miembros de la $\mathrm{CdP}, \mathrm{y}$ mejorar sus habilidades para comprender los problemas de información del usuario final e interactuar con él de manera más asertiva.

En cada uno de los grupos, los sujetos realizaron acciones colectivas e individuales. Por ejemplo, en el caso de los facilitadores, ellos participaron en el diseño de las acciones que se realizaron en el taller. El cuadro 1 muestra una caracterización de las acciones realizadas en cada grupo.

En el siguiente apartado se describen grosso modo los instrumentos utilizados en el este estudio.

\begin{tabular}{|c|c|}
\hline Grupos & Acciones \\
\hline $\begin{array}{l}\text { Grupo 1: } \\
\text { Paraprofesionales. }\end{array}$ & $\begin{array}{l}\text { Compartir conocimientos, } \\
\text { habilidades y experiencias. } \\
\text { Colaborar con el grupo. } \\
\text { Solucionar problemas } \\
\text { relacionados con las estrategias } \\
\text { de búsqueda de información y } \\
\text { las herramientas del catálogo. } \\
\text { Proponer y analizar las ideas. } \\
\text { Tomar decisiones y aplicarlas. } \\
\text { Comunicar información. }\end{array}$ \\
\hline $\begin{array}{l}\text { Grupo 2. Formado } \\
\text { por los Profesionales } \\
\text { a cargo de impartir } \\
\text { el Taller }\end{array}$ & $\begin{array}{l}\text { Diseñar, implementar y evaluar el } \\
\text { taller } \\
\text { Facilitar los procesos de } \\
\text { aprendizaje del grupo } 1 . \\
\text { Compartir conocimientos, } \\
\text { habilidades y experiencias. } \\
\text { Proponer y analizar las ideas. } \\
\text { Colaborar con el grupo. }\end{array}$ \\
\hline $\begin{array}{l}\text { Grupo 3. Formado } \\
\text { por los sujetos que } \\
\text { realizan meta- } \\
\text { análisis de los } \\
\text { procesos. }\end{array}$ & $\begin{array}{l}\text { Diseñar el proceso de ALFIN } \\
\text { Analizar el proceso de } \\
\text { conformación y actuación de los } \\
\text { grupos desde la TA. } \\
\text { Analizar los procesos de los } \\
\text { grupos } 1 \text { y } 2 \text {. }\end{array}$ \\
\hline
\end{tabular}

Cuadro I. Acciones de los Grupos de la Comunidad de Práctica

\subsection{Instrumentos}

- Cuestionario para los "aprendices". Este instrumento incluyó con los siguientes apartados: I. Aspectos generales (adscripción, participación en el módulo, etc), II. Evaluación del taller, III. Evaluación del profesor, IV. Autoevaluación. V. Aprendizaje sobre el uso del catálogo, VI. Utilidad y aspectos positivos o negativos del programa y VII Sugerencias y comentarios.

- Cuestionario para los facilitadores del taller. Este instrumento estuvo integrado por los siguientes rubros: I. Organización del taller; II, Autoevaluación; III. El programa del taller; IV El aprendizaje durante el taller y $\mathrm{V}$. Necesidades de capacitación.

- Taller. El taller tuvo como propósito actualizar los conocimientos y habilidades del personal sobre el uso del catálogo bibliográfico de una biblioteca académica. Los elementos que se consideraron en el taller se muestran en la figura 3 y se adaptaron tomando en consideración la TA y las características de la CdP descritas arriba. 


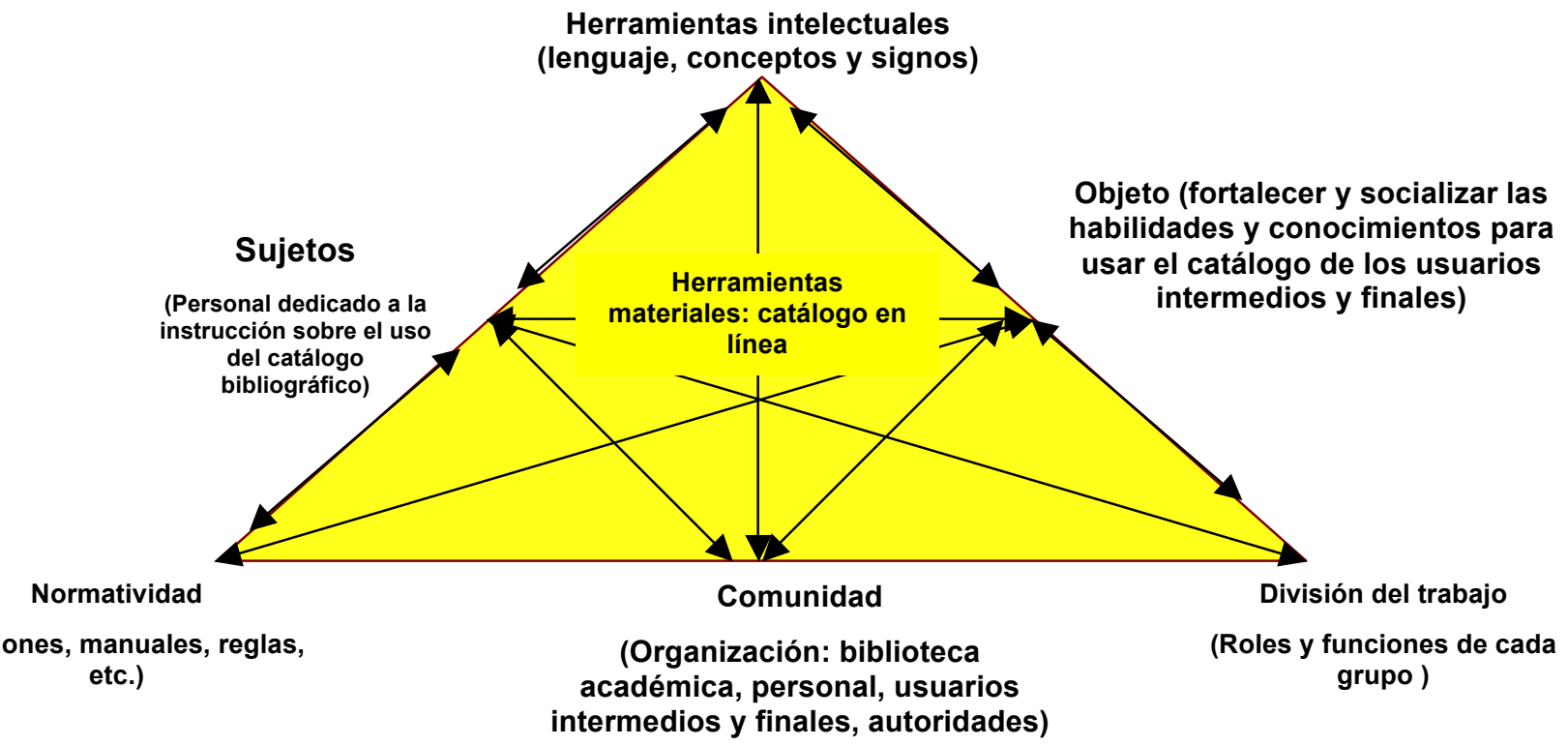

Figura 3. Adaptación de Engeström (1987)

En el taller se buscó evidenciar el catálogo bibliográfico como una herramienta de trabajo y de aprendizaje cotidiano. El taller se llevó a cabo en cinco sesiones de dos horas cada una. Los participantes se dividieron en cuatro grupos, cada uno a cargo de un facilitador. En cada sesión, el facilitador planteó las acciones que tenían que analizar en colaboración. Las acciones que se realizaron fueron las siguientes:

- Acción 1. Análisis de la experiencia del personal. Esta acción consistió en la reflexión grupal en torno a los procedimientos relacionados con la práctica cotidiana en el uso del catálogo de los participantes, quienes debían trabajar en grupos y retomar sus conocimientos y experiencias previas para dar respuesta a 26 preguntas planteadas. Por ejemplo se pidió que establecieran las diferencias entre la búsqueda básica y la avanzada.

- Acción 2. Reflexión sobre los problemas del usuario al buscar en el catálogo. Esta actividad consistió en el análisis de 17 casos reales de búsquedas de información en el catálogo, en las que los usuarios finales obtuvieron cero resultados. Los participantes en el taller debían trabajar en grupos para encontrar la mejor forma de ayudar al usuario en la búsqueda de información. Por ejemplo detectar el problema que un usuarios tenía al buscar una frase o palabra en inglés de la que no conocía su forma de escritura (psychologi, sicology, sycology).

En el taller se hizo énfasis en que era necesario que todos expusieran las operaciones concretas que realizaban en su práctica cotidiana y que escucharan las de los otros. Los facilitadores guiaron cada una de las acciones, a fin de ayudar a los aprendices en la interpretación de sus experiencias. Al finalizar el taller se aplicaron los dos instrumentos. Estos fueron codificados en Excel para construir las tablas descriptivas.

\subsection{Resultados del cuestionario} para aprendices.

De los 62 paraprofesionales que participaron en el estudio, 50 respondieron el cuestionario. Los sujetos que respondieron tuvieron una percepción positiva de su propio desempeño dentro del taller. Al respecto, una mayoría de ellos (85\%) respondieron que, en parte, esto fue motivado por la buena comunicación ya que les permitió preguntar cuando tenían dudas y expresarse libremente. El $90 \%$ de los sujetos consideró que sus participaciones fueron regulares y útiles para realizar las operaciones que les solicitaba el facilitador (45 sujetos).

Todos los participantes en el taller se sintieron motivados ya que pudieron expresar sus puntos de vista y experiencias para resolver los problemas planteados. El trabajo colaborativo para la exposición y resolución de problemas fue 
favorable para el aprendizaje, también fue necesario que algunos temas fuesen expuestos íntegramente por el facilitador. En todo caso, la diversidad de formas de enseñanza se ajustó a la naturaleza del problema, las características del grupo, la capacidad de adaptación del facilitador y a la cantidad de participantes.

Se observó que el $98 \%$ de los sujetos consideró que las interacciones entre pares fueron positivas, el $94 \%$ consideró que fueron respetuosas. El $96 \%$ consideró que los participantes se apoyaron entre sí cuando tuvieron dudas, manteniéndose abiertos a las ideas de los demás $(92 \%)$. En relación al ambiente en el que se desarrolló el taller, el $96 \%$ del grupo consideró que éste fue bueno.

En relación a la autoevaluación de su aprendizaje, el 96\% (48 paraprofesionales) consideró que aprendieron nuevas herramientas de búsqueda de información y que desarrollaron nuevas habilidades para usar el catálogo, por lo que ahora se sienten más eficaces en la tarea. En este mismo sentido, el $98 \%$ de los sujetos consideró que los ejemplos y los ejercicios les ayudaron a comprender mejor los problemas que tienen los usuarios con el catálogo y la forma en la que sus pares resuelven los problemas que se les presentan de manera cotidiana.

\subsection{Resultados de los cuestionarios de los facilitadores}

La siguiente parte de la evaluación estuvo conformada por el cuestionario que se aplicó a los cuatro facilitadores, quienes valoraron de manera positiva el taller.

En general, los facilitadores consideraron que la comunicación y la toma de decisiones en el grupo de facilitadores fueron buenas. La interacción entre el grupo de profesionales y la apertura hacia otras ideas fueron consideradas como adecuadas. En relación a sus aportaciones, tres de los facilitadores consideraron que habían sido muy útiles. Todos consideraron que la interacción uno-a-uno y la apertura hacia otras ideas había sido adecuada. Una persona consideró que el ambiente había sido "Muy bueno" y las otras tres que había sido "Bueno".

También coincidieron en que los participantes habían aprendido y que, inclusive, algunos de ellos habian desarrollado estrategias de búsqueda más elaboradas. Señalaron que la mayoría de los asistentes estuvieron atentos, aunque hubo casos de personas que se mostraban poco interesadas. Otras reacciones positivas de los participantes fueron su apertura al taller y su capacidad para organizarse por sí mismos para aprender. La participación individual también fue espontánea y útil para el desarrollo del taller. De manera importante, se evidenció que era necesario mantener un clima de respeto y de colaboración entre los participantes y el facilitador.

\section{Análisis de resultados y conclusiones}

El estudio alcanzó los objetivos que se planteó. Fue posible analizar la percepción de los integrantes de una CdP sobre la importancia de compartir experiencias y conocimientos para solucionar problemas relacionados con su práctica cotidiana. A partir de ello, fueron capaces de identificarse como miembros de una comunidad que tenia un interés compartido y que no habia tenido la oportunidad de verbalizarlo y socializarlo al interior del grupo. También pudieron identificar quienes tenían mejores estrategias para usar el catálogo e instruir a los usuarios finales sobre el mismo, con la posibilidad de integrar estas estrategias en sus propias acciones.

A partir de los resultados obtenidos en los cuestionarios reportados, podemos identificar que la forma en la que se diseño el taller fue la adecuada para los propósitos planteados. Esto es porque favoreció ambientes de aprendizaje colaborativo en los que los participantes se expresaron abiertamente y discutieron problemas de manera clara y directa, sin mayor preámbulo.

Los ambientes de aprendizaje que favoreció el taller permitieron observar la construcción social del conocimiento entre aprendices y facilitadores, en los términos del aprendizaje situado de Lave y Wenger (1991). En efecto se observó que participantes se percibieron a sí mismos como más hábiles para comprender al usuario final, lo que, por lo menos a este nivel perceptual, nos indica que se sintieron más "expertos". Este es un aspecto al que se dará mayor atención en posteriores estudios.

Otro aspecto importante del taller fue la contextualización del conocimiento y de las habilidades en relación al uso del catálogo. Es decir, se centro en la solución de problemas reales vinculados con el trabajo cotidiano.

En el caso de este estudio, el concepto de comunidades de práctica se complemento con la TA y la ALFIN con el propósito de contar con una estructura teórica y metolodógica fuerte. Dentro de la comunidad los grupos llevaron a cabo procesos de negociación de significados, mismos que les otorgaron el reconocimiento de la identidad del grupo. También se hicieron explícitos los usos del catálogo y se establecie- 
ron reglas no escritas de colaboración y de compromiso mutuo.

Podríamos afirmar que, en el proceso, los sujetos aprendieron a interrelacionarse de mejor manera, ya que, como apuntan los resultados, el nivel de comunicación e interacción entre los paraprofesionales y los profesionales resultó muy bueno. Uno de los factores que influyó es que el curso se planteó como un espacio para compartir experiencias e intercambiar ideas, más que como un espacio de un grupo hegemónico en donde el otro grupo se limitaría a recibir el conocimiento de aquél.

A partir de ello, hacemos las siguientes recomendaciones: 1) apoyar el diseño de sesiones de ALFIN recuperando la práctica cotidiana del personal que interactúa con el catálogo y los usuarios finales e intermedios; 2) trabajar de manera integrada, en un ambiente relajado en el que se fomente el intercambio de ideas y su libre expresión; 3) fomentar el aprendizaje colaborativo; y 4) valorar las contribuciones de todos los participantes en un proceso de ALFIN como esenciales para el desarrollo de una actividad.

En suma, proponemos que los programas de ALFIN se diseñen pensando en actividades que sean significativas para todos los participantes y que estos programas atiendan la creación de ambientes en donde se fomente el aprendizaje mutuo, el respeto y la confianza (tanto entre expertos y novatos, como entre los mismos novatos). Además de ello, que se considere que la ALFIN no se limita a los espacios escolares sino que cada vez está más presente en los ambientes laborales y sociales.

\section{Referencias}

American Library Association. Presidential Committee on Information Literacy, (ALA.PCIL). (1989). Final report, http://www.ala.org/ala/mgrps/divs/acrl/publications/white papers/presidential.cfm. (2010-09-04).

Barab, S., Evans, M. A.,y Baek, E. (2004). Activity theory as a lens for characterizing the participatory unit. En D.H. Jonassen (Ed.), Handbook of research on educational communications and technology. 2a ed. Mahwah, New Jersey: Lawrence Erlbaum Ass 199-214 .

Díaz Barriga Arceo, F. (2003). Cognición situada y estrategias para el aprendizaje significativo. En REDIE: Revista Electrónica de Investigación Educativa. 5:2 105-117. http://redalyc.uaemex.mx/src/inicio/ArtPdfRed.jsp?iCve= 15550207\# (2010-09-04).

Bawden, D. (2002). Revisión de los conceptos de alfabetización informacional y alfabetización digital. // Anales de Documentación. 5 (2002) 361-408.

Bereiter, C. y Scardamalia, M. (1996). Rethinking learning. // Olson, D.; Torrance, N. (Eds.). The Handbook of Education and Human Development. (485-513). Cambridge : Blackwell

Bilal, D. (2004). Research on children's information seeking on the Web. // Chelton, M. K.; Cool, C. (Eds.). Youth in- formation-seeking behaviour: Theories and issues (pp. 271-291). Lanham, Maryland: The Scarecrow Press.

Courtight, C. (2007). Context in information behavior research. // Annual Review of Information Science and Technology. 41 (2007) 273-306.

Dalkir, K. (2005) Knowledge management in theory and practice. Boston, MA: Elsevier Butterworth Heinemann, 2005.

Davenport E. y Hall, H. (2002). Organizational knowledge and communities of practice. // Annual Review of Information Science and Technology. 36 (2002) 171-227.

Díaz Barriga, F. y Hernández, G. (2002). Estrategias docentes para un aprendizaje significativo: una interpretación constructivista. México, D.F.: McGrawHill.

Eisenberg, M. (2004). Effective users of information on standardized tests. Library Media Connection. http://www.galeschools.com/pdf/Eisenberg.pdf (2010-0904).

Engeström, Y. (1987). Learning by expanding: An activitytheoretical approach to developmental research. Helsinki: Orienta-Konsultit.

Eyre, G. (2001). The role of works of imagination in preparing young people for the information society. // Libraries and Librarians: Making a Difference in the Knowledge Age. Council and General Conference: Conference Programme and Proceedings, Boston, MA. (ERIC Clearinghouse on Information Resources, Service No. ED459717).

Foster, A. (2004). A nonlinear model of information-seeking behaviour. // Journal of the American Society for Information Science and Technology. 55:3 (2004) 228-245.

Foster, A. (2006), Students fall short on information literacy: Educational testing service's study finds. // The Chronicle of Higher Education, 53 (2006) 27.

Gracy, K.F. (2001). Documentar comunidades: el caso de la etnografía archivística. // Nuevos métodos de investigación en archivística. 211-244.

Hjørland, B. (1997). Information seeking and subject representation. An activity-theoretical approach to information science. Westport, CT; London: Greenwood Press.

Johnston, B. y Webber, S. (2005). As we may think: Information literacy as a discipline for the information ages. // Research Strategies. 20(3) (2005) 108-121.

Lau, J. (2007). Latin America information literacy state of the art report. // Lau, J. (Ed.), Informational state of the art report. http://www.jesuslau.com/docs/publicaciones /doc2/UNESCO_state_of_the_art.pdf (2007-05-04).

Lave, J. (1991). Situation learning: legitimate peripheral participation. Cambridge: Cambridge University Press.

Lehman, K. y Dudley, J. (2001). Collaboration in information literacy. // Knowledge Quest. 30:1 ( 2001) 24-25

Leontiev, A. N. (1993/1977). Actividad, conciencia y personalidad. México, D.F.: Asbe.

Lloyd, A. (2005). Information literacy landscapes: an emerging picture. $62: 5$ (2005) 570-583.

Loyarte, E. y Rivera, O. (2007) Communities of practice : a model for their cultivation. // Journal of Knowledge Management. 11:3 (2007) 67-7.

Niemeyer, B. (2004). Situated learning for social and vocational integration in Germany. // Evans, K.; Niemeyer, B. (Eds.), Reconnection: Countering social Exclusion Trough Situated Learning. Dordrecht, NL, S. 47-62

Niemeyer, B. (2006). El aprendizaje situado una forma de escapar del enfoque de déficit. // Revista de Educación. 341 (Septiembre-diciembre 2006) 99-121.

Ortiz, V. Vega, G. y Quijano, A. (2008). Evaluación de la conducta informativa en profesionales de la información. 
// Coloquio sobre Administración y Liderazgo en el Campo Informativo, 21-23 de septiembre del 2008. Veracruz, Ver.

Palincsar, A. S. (1998). Social constructivism perspectives on teaching and learning. // Annual Review of Psychology. 49 ( 1998) $345-375$.

Rader, H.B. (2002). Information literacy, 1973-2002: A selected literature review. // Library Trends. 51:2 (2002) 242261.

Rendon, N., Naranjo, E., y Giraldo, C. (2005). Evolución y tendencias de la formación de usuarios en el contexto de la investigación. // Revista Interamericana de Bibliotecología. 28 (2005) 43-86.

Trigg, P. \& John, P. (2004). From to transformation : information and Communications technology, professional development and the formation of communities of practice. // Journal of Computer Assisted Learning, 20 (2004) 426439.

Vega, G. (2009) Alfabetización informacional: estudio sobre su apropiación con alumnos de primaria. (Tesis doctoral, UNAM, Facultad de Psicología).

Quijano, A. y Vega G. (2008). A theoretical model in order to represent academic libraries as information systems. // 8th International Conference on Sociocybernetics : Complex social systems, interdisciplinarity and world futures [CD-ROM]. Ciudad de México, México.

Vygotsky, L. S. (1978). Mind in society: The development of higher psychological processes. Cambridge, Ma.: Harvard University Press.

Wastawy, S. (2004). Learning communities: a fundamental shift in the learning process, an investigative study into their impact in library services. En: 24th IATUL Conference, Libraries and Education in the Networked Information Environment (2-3 June, 2003). http://www.iatul.org/doclibrary/public/Conf_Proceedings/ 2003/WASTAWY_fulltext.pdf (2010-09-04).

Wilson, T. D. (2000). Human information behavior. // Informing Science. 3:3 (2000) 49-55.

Wilson, T. D. (2008). Activity theory and information seeking. // Annual Review of Information Science and Technology, 42 (2008) 119-161.

Wenger. E. (1998/2001). Comunidades de práctica: aprendizaje, significado e identidad. Barcelona, España: Paidós.

Yates, J. y Orlikowski, W. (1992). Genres of organizational communication: a structuration approach to studying communication and media. // Academy of Management Review, 17:2 (1992) 299-326.

Recibido: 1010-07-16. Revisado: 2010-07-16.

Aceptado: 2010-09-03 
